J. Lake Sci.(湖泊科学), 2017, 29(4): 819-826

DOI 10. 18307/2017. 0405

(C) 2017 by Journal of Lake Sciences

\title{
湖泊营养盐通量平衡的三维数值模拟”
}

\author{
邹 锐 ${ }^{1,3}$, 吴 桢 ${ }^{2}$, 赵 磊 ${ }^{1}$, 陈异晖 ${ }^{1}$, 余艳红 ${ }^{1}$, 刘 永 $^{2 * *}$ \\ (1: 云南高原湖泊流域污染过程与管理重点实验室, 昆明 650034) \\ ( 2 : 北京大学环境科学与工程学院, 水沙科学教育部重点实验室, 北京 100871) \\ (3:Tetra Tech, Inc. 10306 Eaton Place, Ste 340, Fairfax, VA 22030, USA)
}

\begin{abstract}
摘 要: 湖泊氮、磷通量是表征湖泊营养状态的重要指标, 也是探究湖泊富营养化机制的重要途径. 通过氮、磷通量的计 算和质量平衡关系的分析, 可以识别出在湖泊富营养化过程中起关键作用的过程. 以三维水动力-水质模型为计算平台, 模拟湖泊水动力、水质的动态过程, 并以模拟结果为基础核算湖泊氮、磷循环通量及其在氮、磷循环整体中的贡献, 识别 湖泊氮、磷循环关键源汇过程的变化规律. 滇池是我国富营养化湖泊的代表, 同时其半封闭特性为营养物质循环提供了 更为稳定的环境, 以滇池为案例,基于前期校正和验证过的滇池水动力-水质模型来分析循环通量计算方法的适用性. 结 果发现, 研究年度内滇池外海总氮的年总输人量 (包括外源和内源) 为 $7620.92 \mathrm{t}$, 总输出量 (包括出流、反硝化和沉降) 为 $7637.31 \mathrm{t}$; 总磷的年总输入量为 (包括外源和内源) $450.23 \mathrm{t}$, 总输出量 (包括出流和沉降) 为 $429.57 \mathrm{t}$, 其中陆域输人是最主 要的氮、磷输人途径, 而反硝化和沉降则是主要的输出过程. 相较于传统的质量平衡方法,通过三维模型计算所得的营养 盐通量平衡结果能更好地揭示湖体内所发生的氮、磷转化过程.
\end{abstract}

关键词: 营养盐; 通量;质量平衡;三维模型;滇池

\section{Nutrient cycling flux of Lake Dianchi: A three-dimensional water quality modelling ap- proach}

\author{
ZOU Rui ${ }^{1,3}$, WU Zhen ${ }^{2}$, ZHAO Lei ${ }^{1}$, CHEN Yihui ${ }^{1}$, YU Yanhong ${ }^{1}$ \& LIU Yong ${ }^{2 * *}$ \\ (1: Yunnan Key Laboratory of Pollution Process and Management of Plateau Lake-Watershed, Kunming 650034, P.R. China) \\ (2: College of Environmental Science and Engineering, The Key Laboratory of Water and Sediment Sciences Ministry of Educa- \\ tion, Peking University, Beijing 100871, P.R.China) \\ (3: Tetra Tech, Inc.10306 Eaton Place, Ste 340, Fairfax, VA 22030, USA)
}

\begin{abstract}
Nutrient cycling flux in lakes is an important indicator to measure the lake trophic state as well as an essential approach to explore eutrophication mechanisms. Key processes of nutrient cycling can be identified by calculating nutrient cycling fluxes and mass balance analysis. In this study, IWIND-LR, a 3D hydrodynamic and water quality model, was used to simulate nutrient cycling of lakes in this study. The nutrient cycling fluxes were calculated based on the model results to define the contributions and patterns of nutrient source and sink processes. Lake Dianchi in the Yunnan Plateau is one of the most severely polluted lakes in China. It is a semi-closed lake and provides a stable environment for research. The model results demonstrated that, IWIND-LR performed well in Lake Dianchi. The input and output of total nitrogen in Lake Dianchi was $7620.92 \mathrm{t}$ and $7637.31 \mathrm{t}$ respectively in 2003; while $450.23 \mathrm{t}$ and $429.57 \mathrm{t}$ for total phosphorus. The exogenous loading contributed most of the input of nitrogen and phosphorus, while denitrification and sedimentation were the major outputs of Lake Dianchi.
\end{abstract}

Keywords: Nutrient cycling; flux; mass balance; Three-Dimensional Modeling; Lake Dianchi

湖泊富营养化是水环境领域面临的突出问题 ${ }^{[1-4]}$. 为有效预防、控制和治理湖泊富营养化问题, 国内外

* 国家重点基础研究发展计划“973”项目(2015CB458900)和云南省高原湖泊流域污染过程与管理重点实验室开发 基金(2015) 联合资助. 2016-03-25 收稿; 2016-10-12 收修改稿. 邹锐 (1968 ), 男, 博士, 研究员; E-mail: rz5q2008@ gmail.com.

** 通信作者; E-mail:yongliu@ pku.edu.cn. 
围绕其成因、危害以及控制和管理等方面开展了大量的研究 ${ }^{[5-10]}$, 其中成因分析是有效治理的基础. 研究表 明,氮 $(N)$ 、磷 $(P)$ 是湖泊生态系统必需的营养元素, 正常的氮、磷平衡是维持湖泊健康的必要条件; 但大量 氮、磷的流入和累积会改变湖泊内营养盐的通量平衡, 增加富营养化的风险 ${ }^{[1-13]}$. 因此对湖泊恢复而言, 开 展湖泊氮、磷营养盐循环和通量平衡研究就显得尤为重要 ${ }^{[9,14-15]}$. 具体而言, 氮、磷的过量输人会导致湖泊营 养盐循环发生改变, 藻类大量生长、消亡, 影响水体的理化条件并进一步改变氮、磷的内部循环过程, 增加湖 泊富营养化的风险 ${ }^{[16]}$. 研究表明, 随着湖泊外源污染负荷的削减, 湖泊底质内源释放对湖泊富营养化的贡 献也逐渐突显出来 ${ }^{[17]}$. 氮、磷的内源释放和内部循环会使湖泊在大量削减外源负荷后, 也继续维持长时间 的富营养化状态, 阻碍湖泊修复. 研究发现, 对于部分富营养化的湖泊, 内源负荷可以占到总人湖负荷量的 $50 \%$ 以上 ${ }^{[18]}$. 同时,一些富营养化湖泊的修复过程表明: 在有效控制外源负荷的前提下, 仍需要 $10 \sim 20$ 年才 能使湖泊的生态功能得到明显的恢复 ${ }^{[19]}$. 因此, 通过对湖泊氮、磷循环通量的追踪, 可分析湖体中所发生的 氮、磷迁移转化过程,识别不同氮、磷循环过程的响应机制, 加深对富营养化过程及机理的认知. 机理模型是 研究湖泊富营养化过程和营养盐通量平衡的理想手段 ${ }^{[20-21]}$, 通过对水体中与富营养化相关机理过程的模 拟,为制定更为细致和准确的治理方案提供科学依据. 在模型的应用中, 简单机理模型过程简单, 无法实现 对重要营养盐过程的刻画; 而复杂的三维水动力-水质模型研究则大多关注于模型的性能及其不确定性, 对 氮、磷循环过程的关注较少 ${ }^{[22]}$.

本文基于以 EFDC 为内核的三维水动力 -水质模型 (IWIND-LR), 开发了利用数值积分的氮、磷营养盐 通量与存量计算方法, 并以滇池外海为例开展应用研究. 滇池 $\left(24^{\circ} 40^{\prime} \sim 25^{\circ} 02^{\prime} \mathrm{N}, 102^{\circ} 36^{\prime} \sim 102^{\circ} 47^{\prime} \mathrm{E}\right)$ 是我国 重点治理的“三湖”之一, 湖面面积为 $306.3 \mathrm{~km}^{2}$, 湖体体积为 $15.6 \times 10^{8} \mathrm{~m}^{3}$, 平均水深 $4.4 \mathrm{~m}$, 流域面积 2920 $\mathrm{km}^{2[23]}$. 由于流域社会经济的快速发展, 滇池从 1980s 开始出现富营养化现象, 目前呈重度富营养状态; 外海 水质在 $\mathrm{V} \sim$ 劣 $\mathrm{V}$ 类之间波动, 且呈现北部偏高、南部偏低的趋势 ${ }^{[24]}$. 本文基于前期经过校正和验证的滇池三 维水动力-水质模型 ${ }^{[23]}$, 在此基础上重点开展氮、磷的内部循环过程通量研究, 分析滇池水质和藻类生物量 的变化过程, 识别不同氮、磷循环过程对湖泊富营养化的贡献, 为滇池富营养化治理提供科学依据.

\section{1 研究方法}

\section{1 模型构建}

模型构建基于前期经过校正和验证的滇池三维水动力 -水质模型 ${ }^{[23]}$, 主要包含三维水动力和温度动力 学模拟以及内部耦合的水质模型系统; 可模拟 26 个水质状态变量, 并可同时模拟底泥与水体间的营养盐交 换动力学以及底泥对溶解氧的动态作用, 动态跟踪水龄及低溶解氧区的时空分布. 其核心的水质模块主要 基于物质守恒, 刻画物理输送、大气交换、吸附与解吸、藻类吸收、底床-水界面交换、硝化与反硝化以及沉积 成岩作用等,基本控制方程如下:

$$
\frac{\partial C}{\partial t}+\frac{\partial(u C)}{\partial x}+\frac{\partial(v C)}{\partial y}+\frac{\partial(w C)}{\partial z}=\frac{\partial}{\partial x}\left(K_{x} \frac{\partial C}{\partial x}\right)+\frac{\partial}{\partial y}\left(K_{y} \frac{\partial C}{\partial y}\right)+\frac{\partial}{\partial z}\left(K_{z} \frac{\partial C}{\partial z}\right)+S_{c}
$$

式中, $C$ 为水质状态变量浓度, $u 、 v 、 w$ 分别为 $x 、 y 、 z$ 方向的速度矢量, $K_{x} 、 K_{y} 、 K_{z}$ 分别为 $x 、 y 、 z$ 方向的湍流扩散 系数, $S_{c}$ 为每单位体积内部和外部的源和汇. 在一阶动力学中, 存在以下关系:

$$
S_{c}=k \cdot C+R
$$

式中, $k$ 为动力学速率, $R$ 为由于外部负荷和内部反应引起的源汇项.

在前期的滇池三维水动力-水质模型 ${ }^{[23]}$ 构建中, 将滇池外海水体划分为垂向 6 层, 每层 664 个水平网格 ( 尺度为 $634 \pm 148 \mathrm{~m}$ ) (图 1), 主要模拟的状态变量为水温、溶解氧、藻类中氮磷含量、颗粒态有机氮磷浓度、 溶解态有机氮磷浓度、磷酸盐浓度、硝态氮浓度、氨氮浓度等. 模型的边界条件主要包括人湖支流的流量、温 度、水质、沉积床初始条件以及与时间相关的气象条件和大气边界数据 ${ }^{[23]}$, 其中大气湿沉降条件为磷酸盐浓 度 $0.04 \mathrm{mg} / \mathrm{L}$, 硝态氮浓度 $0.70 \mathrm{mg} / \mathrm{L}$. 模型底泥模块初始条件为: 活性有机磷、有机氮和有机碳底泥体积浓 度分别为 $23 \pm 15.3 、 103.2 \pm 13.8$ 和 $741 \pm 176.5 \mathrm{~g} / \mathrm{m}^{3}$.

\section{2 营养盐存量与通量核算}

全湖氮、磷营养盐各组分的存量和通量计算模块是通过与三维水质模型方程耦合, 并与水动力-水质模 


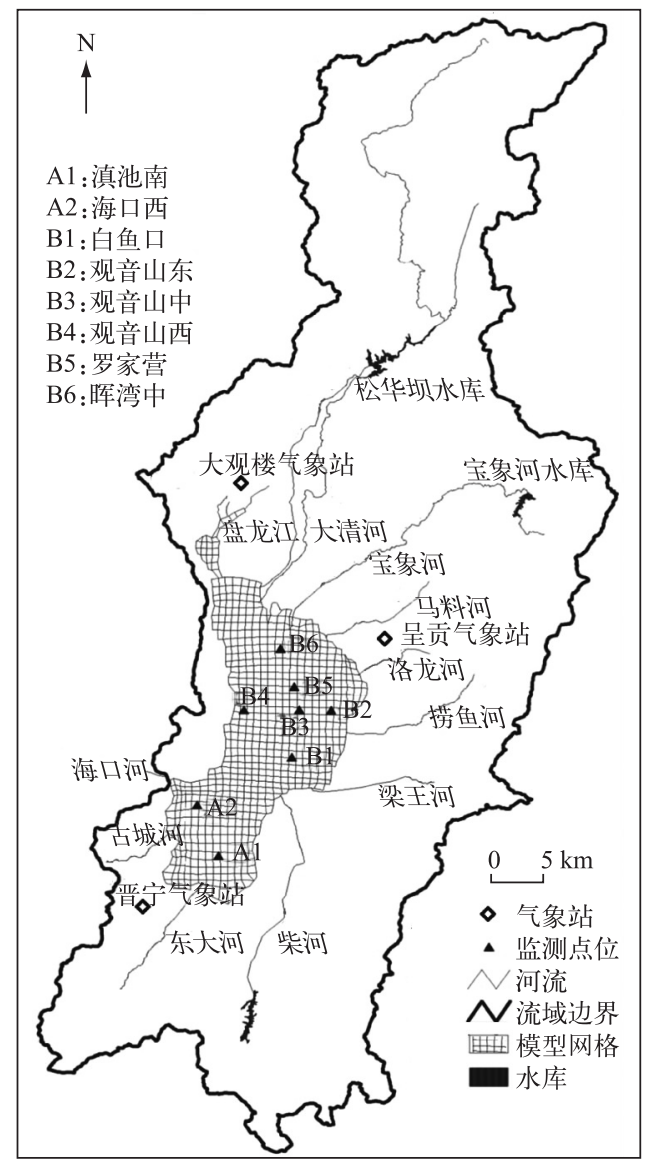

图 1 滇池三维水动力-水质模型的平面网格划分 ${ }^{[23]}$

Fig.1 Grid division of IWIND-LR of Lake Dianchi

型采用相同离散化分辨率进行数值积分得到的. 各组分存量考虑水质组分的时空分异性, 各相关状态变量 的存量通过对全湖所有网格进行积分获取:

$$
M(i, t)=\iiint c(i) \mathrm{d} x \mathrm{~d} y \mathrm{~d} z
$$

式中, $M$ 为 $i$ 组分的存量, $c$ 为湖体中 $i$ 组分的浓度. 氮元素各组分存量考虑: 藻类活体氮存量 (algaeN)、颗粒 有机氮存量 $(\mathrm{PON})$ 、溶解态有机氮存量 $(\mathrm{DON})$ 、氨氮存量 $\left(\mathrm{NH}_{3}\right)$ 、硝酸盐存量 $\left(\mathrm{NO}_{3}^{-}\right)$; 磷元素各组分存量考 虑: 藻类活体磷存量 $\left(\right.$ algaeP)、颗粒有机磷存量 $(\mathrm{POP})$ 、溶解态有机磷存量 $(\mathrm{DOP})$ 、正磷酸盐存量 $\left(\mathrm{PO}_{4}\right)$.

各氮、磷循环过程通量通过对全湖所有网格进行积分获取, 氮循环各个过程通量考虑以下过程: (1)流域 输人 (包括藻类活体氮人湖量、颗粒有机氮人湖量、溶解态有机氮人湖量、氨氮人湖量、硝酸盐/亚硝酸盐氮 人湖量) ; (2)大气沉降 (包括硝酸盐氮沉降) ; (3)出流 (包括藻类活体氮出湖量、颗粒有机氮出湖量、溶解态有 机氮出湖量、氨氮出湖量、硝酸盐/亚硝酸盐出湖量) ; (4)内源 (包括氨氮底泥交换通量、硝酸盐氮底泥交换通 量); (5)沉降 (包括藻类活体氮沉降量和颗粒有机氮沉降量); (6) 反硝化过程和固氮. 磷循环各个过程通量考 虑以下过程: (1) 流域输人 (包括藻类活体磷人湖量、颗粒有机磷人湖量、溶解态有机磷人湖量、正磷酸盐入湖 量) ; (2)大气沉降 (包括正磷酸盐沉降组分) ; (3)出流 (包括藻类活体磷出湖量、颗粒有机磷出湖量、溶解态有 机磷出湖量、正磷酸盐出湖量) ; (4)内源 (包括正磷酸盐底泥交换通量) ; (5)沉降 (包括藻类活体磷沉降量、颗 粒有机磷沉降量、正磷酸盐沉降量).上述过程的主要通量计算方程如下: 
流域输人通量:

累积大气沉降通量:

$$
F_{\mathrm{WS}(i)}=\sum_{i=t_{0}}^{T} \sum_{k=1}^{N} Q(t, k) \cdot C(t, k, i)
$$

$$
F_{\text {AIR }(i)}=\iiint\left(D_{\mathrm{d}}+D_{\mathrm{w}}\right) \mathrm{d} x \mathrm{~d} y \mathrm{~d} t
$$

累积出流通量:

累积底泥-水体通量:

$$
F_{\mathrm{O}(i)}=\sum_{k=1}^{M} \int q(k, t) c(k, t) \mathrm{d} t
$$

$$
F_{\operatorname{Ben}(i)}=\iiint F_{\mathrm{b}(i)} \mathrm{d} x \mathrm{~d} y \mathrm{~d} t
$$

累积沉降通量:

$$
F_{\mathrm{Set}(i)}=\iiint F_{\mathrm{s}(i)} \mathrm{d} x \mathrm{~d} y \mathrm{~d} t
$$

累积反硝化通量:

$$
F_{\text {Den }}=\iiint \int F_{\mathrm{dn}} \mathrm{d} x \mathrm{~d} y \mathrm{~d} z \mathrm{~d} t
$$

累积固氮量:

$$
F_{\text {Nfix }}=\iiint \int F_{\text {Nfix }} \mathrm{d} x \mathrm{~d} y \mathrm{~d} z \mathrm{~d} t
$$

式中, $Q$ 为人湖流量 $\left(\mathrm{m}^{3} / \mathrm{d}\right), C$ 为每条人湖河流中 $i$ 组分的浓度 $\left(\mathrm{g} / \mathrm{m}^{3}\right), D_{\mathrm{d}}$ 为干沉降 $\left(\mathrm{g} /\left(\mathrm{m}^{2} \cdot \mathrm{d}\right)\right), D_{w}$ 为湿 沉降 $\left(\mathrm{g} /\left(\mathrm{m}^{2} \cdot \mathrm{d}\right)\right), q$ 为出流流量 $\left(\mathrm{m}^{3} / \mathrm{d}\right), c$ 为出流中 $i$ 组分的浓度 $\left(\mathrm{g} / \mathrm{m}^{3}\right), F_{\mathrm{b}}$ 为 $i$ 组分的底泥释放速率 $\left(\left(\mathrm{g} /\left(\mathrm{m}^{2} \cdot \mathrm{d}\right)\right), F_{\mathrm{s}}\right.$ 为 $i$ 组分的沉降速率 $\left(\mathrm{g} /\left(\mathrm{m}^{2} \cdot \mathrm{d}\right)\right), F_{\mathrm{dn}}$ 为反硝化速率 $\left(\mathrm{g} /\left(\mathrm{m}^{3} \cdot \mathrm{d}\right)\right), F_{\mathrm{Nfix}}$ 为固氮速率 $(\mathrm{g} /$ $\left.\left(\mathrm{m}^{3} \cdot \mathrm{d}\right)\right)$. 需要注意的是, 根据以上方程计算的氮、磷循环通量结果并不是瞬时通量结果, 而是累积通量结 果. 为计算湖泊氮、磷循环通量和存量, 模型以相同条件连续运行 5 年以达到相对稳定状态, 取第 5 年结果 计算湖泊氮、磷循环通量及存量.

\section{2 研究结果}

\section{1 模型校验}

模型校验是水质模型构建的核心步骤, 目的是推算合理的参数化方案以实现模型对实际湖体过程的再 现 ${ }^{[25]}$. 滇池模型的校验分 2 个步骤: 水动力模型的校验、水质模型校验; 其中水动力模型以水位和温度作为 评判标准, 水质模型校验以滇池外海 8 个国家常规监测点的月监测数据, 主要校准溶解氧 (DO)、总氮 (TN)、 总磷 (TP)、 $\mathrm{NH}_{3}-\mathrm{N}$ 、叶绿素 a (Chl.a) 浓度等. 由于本文采用的是前期经过校正和验证的模型, 限于篇幅, 其校 正和验证在此不再赘述,具体结果请参阅相关文献 ${ }^{[23]}$. 由校正和验证结果可知 ${ }^{[23]}$, TN、TP 和 Chl. a 的模拟 结果与实测值吻合较好, RMSE 分别为 $0.619 、 0.064$ 和 $0.040 \mathrm{mg} / \mathrm{L}$, 模型能够较为真实反映湖体物理、化学和 生物过程的变化. 校核后的模型参数取值如下: 水体最大硝化反应速率为 $0.05 \mathrm{~d}^{-1}$, 参照温度是 $25^{\circ} \mathrm{C}$, 温度效 应系数为 0.045 ; 反硝化速率为 $0.06 \mathrm{~d}^{-1}$; 有机氮矿化速率为 $0.05 \mathrm{~d}^{-1}$, 有机磷矿化速率为 $0.04 \mathrm{~d}^{-1}$, 有机碳为 $0.06 \mathrm{~d}^{-1}$, 矿化速率温度修正系数为 0.069 ; 颗粒营养盐沉降速率为 $0.1 \mathrm{~m} / \mathrm{d}$; 蓝藻、硅藻和绿藻生长速率分别 为 $1.35 、 2.5$ 和 $1.9 \mathrm{~d}^{-1}$. 底泥通量采用底泥成岩模块计算, 主要参数为: $\mathrm{G} 1$ 类有机物降解速率为 $0.015 \mathrm{~d}^{-1}, \mathrm{G} 2$ 类为 $0.001 \mathrm{~d}^{-1}, \mathrm{G} 3$ 类为 0 , 温度修正系数为 1.09 ; 底泥硝化最佳反应速率为 $0.09 \mathrm{~d}^{-1}$.

\section{2 滇池营养盐通量存量平衡核算}

在模型校准的基础上, 对滇池不同形态氮、磷营养盐的存量及主要源汇过程的通量进行计算 (图 2). 由 结果可知, 氨氮、硝态氮和有机氮之间存在明显的相互转化关系和季节特征; 磷酸盐是磷元素各个组分中存 量最大的组分, 呈现明显的夏季增加、冬季减少的趋势. 氮、磷的底泥释放也存在明显的季节变化, 其释放通 量都表现为夏季较大、冬季较低的规律, 其中氨氮和磷酸盐在冬季为负值, 即底泥的吸收过程. 反硝化的季 
节特征与其他过程不同, 7 月之前的反硝化通量明显小于之 后的反硝化通量.

\section{3 关键源汇过程识别}

表 1 汇总了 2003 年滇池湖体氮、磷循环各个过程通量的 年均值, 以 TN 和 TP 计, 滇池 TN 的年总输人量为 $7620.92 \mathrm{t}$, 总输出量为 $7637.31 \mathrm{t}$; TP 的年总输人量为 $450.23 \mathrm{t}$, 总输出 量为 $429.57 \mathrm{t}$. 对于氮而言, 贡献最大的源过程是陆域流人 过程, 占总流人的 $67.76 \%$, 其次是底泥释放过程, 氨氮和硝 态氮共占 $29.04 \%$, 大气沉降和固氮作用共占 $3.2 \%$. 氮的去 除过程则主要为反硝化和沉降, 共占总去除量的 $95.5 \%$, 其 中反硝化 $48.99 \%$, 沉降为 $46.51 \%$. 对于磷而言, 陆域流人过 程占总流人量的 $79.77 \%$, 其次是磷酸盐底泥释放作用, 为 $18.3 \%$. 磷去除则主要为沉降作用, 其中颗粒态磷沉降占 $62.77 \%$, 藻类沉降占 $27.87 \%$.

\section{3 讨论}

\section{1 数值模拟与基于监测的通量核算结果的差异}

首先需要明确的是本文中的营养盐质量平衡关系是基 于三维复杂水动力-水质模型的模拟结果, 是对全湖所有网 格进行数值积分所得到的. 这不同于一般的营养盐质量平 衡分析方法,一般意义上的营养盐质量分析方法是基于有限 的监测数据计算得到的, 在空间上并不能做到与三维模型模 拟结果一样细致, 这也就导致 2 种方法所计算的营养盐质量 平衡会有一定的差异 ${ }^{[22]}$. 虽然营养盐质量平衡分析在更大 的程度上是一种估算, 并不要求观测数据的空间水平和垂直 分布, 但对于面积较大或深度较大的湖泊而言, 会造成结果 的明显偏差. 而如果借助于三维复杂水质模型, 在监测数据 的基础上,对全湖各个网格进行积分后再计算营养盐质量平 衡关系就会避免不必要的偏差. 以滇池为例, 根据对 8 个湖 体常规监测点位的数据, 计算滇池 TP 存量为 $231.4 \mathrm{t}$, TN 存 量为 $3076 \mathrm{t}$; 而通过水动力 -水质模型计算可知, 滇池的 TP 和 TN 存量分别为 350.2 和 $2525.4 \mathrm{t}$, 二者之间均有较大的差 异, 计算结果 TP 偏高、TN 略低. 由国家标准监测方法可知, 所检测的样品均取自水面下 $0.5 \mathrm{~m}$, 其监测数据主要表征的 是表层水体的水质状态. 对于滇池而言, 特别是磷元素, 其 主要来源之一是底泥中磷酸盐的释放, 这会造成湖体磷酸盐 形成自下而上的浓度梯度 ${ }^{[26-28]}$. 这种湖体垂向浓度梯度的 存在会导致在只用表层水质数据估算营养盐通量平衡时产 生系统性误差 (图 3). 除此之外, 考虑到湖泊复杂的水动力 条件以及监测点位的分布并不能完全代表全湖的空间差异, 这也会在估算时造成一定的误差. 相比而言, 基于三维水质 模型的营养盐通量平衡计算则会涉及到全湖所有已划分的 网格, 在模型已校准的情况下, 比传统的估算方法更为精确, 且能提供更为详细的通量结果.
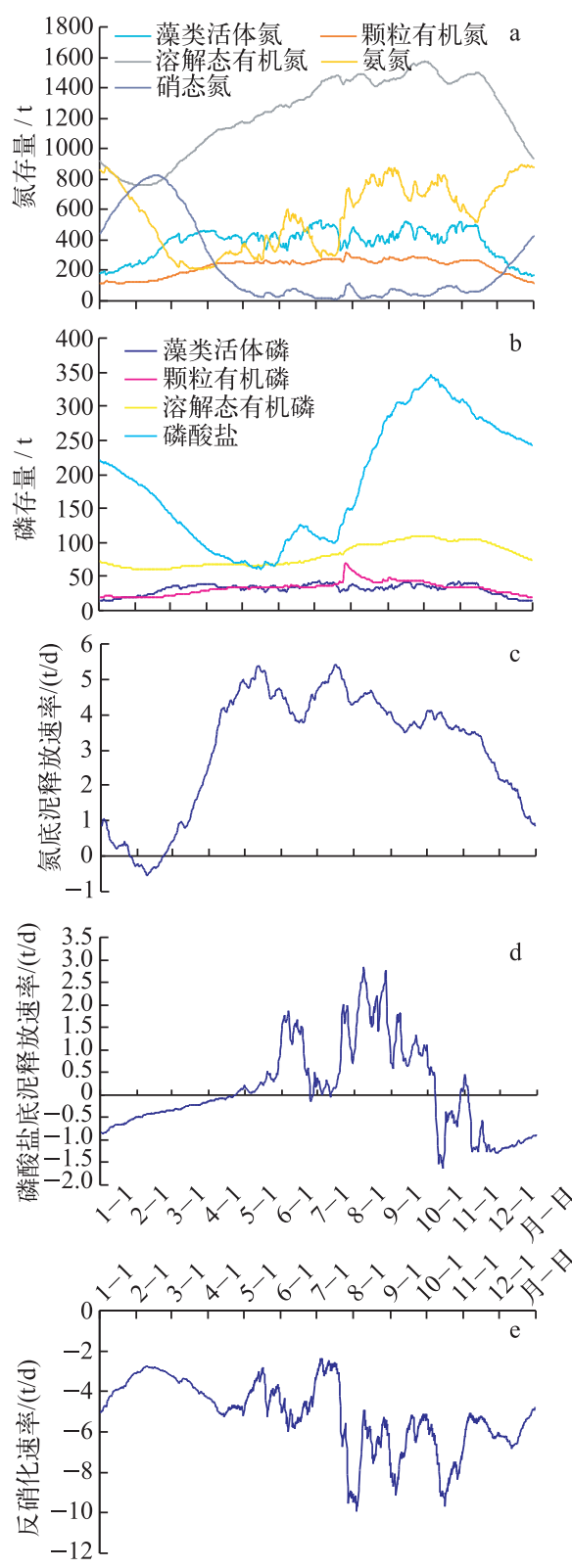

图 2 氮存量 $(a)$ 、磷存量 $(b)$ 、氮底泥释 放速率 $(\mathrm{c})$ 、磷酸盐底泥释放速率 $(\mathrm{d})$ 和 反硝化速率 $(\mathrm{e})$ 变化趋势

Fig. 2 Variation trend of $\mathrm{N}$ amount(a), $\mathrm{P}$ amount(b) sediment release flux of $\mathrm{N}(\mathrm{c})$ sediment release flux of $\mathrm{P}(\mathrm{d})$ and denitrification flux $(\mathrm{e})$ 

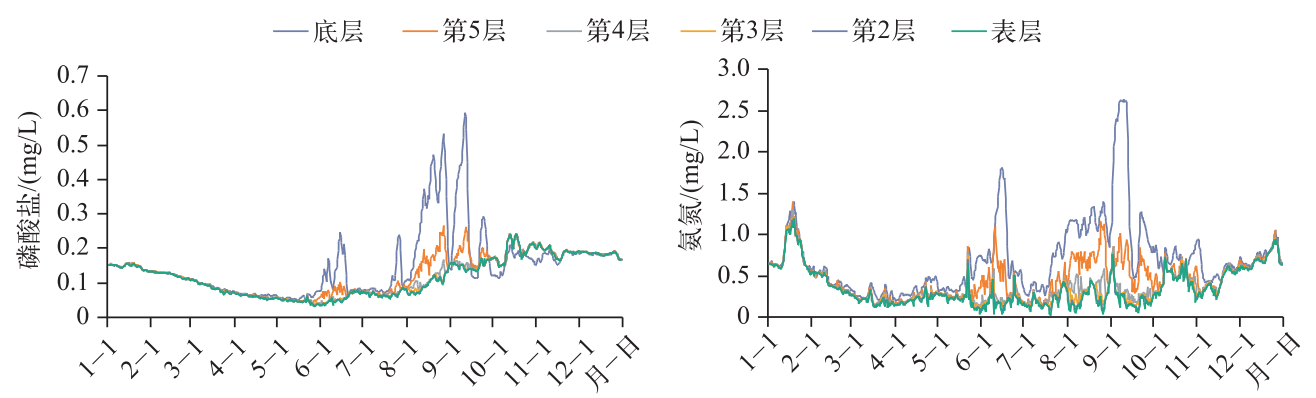

图 3 滇池 B6 站点不同深度的磷酸盐和氨氮浓度变化趋势

Fig.3 Variation trends of phosphate and ammonia concentrations with different depths at site B6 in Lake Dianchi

表 1 滇池氮、磷年存量和通量的平衡关系

Tab.1 N and P storage and cycling fluxes in Lake Dianchi

\begin{tabular}{clcc}
\hline \multirow{2}{*}{} & 主要过程 & \multicolumn{2}{c}{ 通量/t } \\
\cline { 3 - 4 } & & $\mathrm{TN}$ & $\mathrm{TP}$ \\
\hline \multirow{2}{*}{ 输人 } & 大气沉降 & 152.20 & 8.70 \\
& 固氮 & 91.37 & - \\
& 陆域输人 & 5164.29 & 359.14 \\
& 氮底泥释放 & 2213.05 & - \\
& 磷酸盐底泥释放 & - & 82.39 \\
输出 & 出湖河流输出 & 344.00 & 40.19 \\
& 反硝化 & 3741.25 & - \\
& 颗粒有机态沉降 & 2102.02 & 269.65 \\
& 藻类沉降 & 1450.04 & 119.73 \\
\hline
\end{tabular}

\section{2 滇池氮、磷营养盐存量变化}

对湖体内氮、磷元素的存量分析可知, 不同 形态的氮或磷在时间和通量上的变化趋势各 异,具体表现在:

(1)氨氮和硝态氮的变化趋势不同步. 在春 季, 溶解氧浓度相对较高, 随着氨氮浓度的降 低, 硝态氮浓度经历了先升高后降低的过程, 这 主要是因为在有氧环境下氨氮通过硝化作用可 以生成硝态氮. 而夏季由于藻类的大量生长甚 至暴发, 水体中溶解氧浓度降低, 硝态氮会通过 反硝化作用生成氮气逸出湖体 ${ }^{[29-30]}$. 在 7 月中下 旬藻类暴发后, 反硝化作用显著增强 (图 $2 \mathrm{e}$ ), 同 时缺氧环境也抑制了硝化作用, 促进了底泥释 放氨氮的过程, 所以硝态氮在夏季保持较低浓 度, 而氨氮由于底泥释放作用和藻类吸收的共同作用会出现较大的波动. 秋、冬季则由于藻类生物量降低, 水体溶解氧浓度升高会使水体内氨氮和硝态氮浓度逐渐升高.

(2)水体中颗粒态有机氮和溶解态有机氮浓度比例在 $1: 5$ 左右, 且变化趋势大致相同, 这符合湖沼学基本 定律中的描述 ${ }^{[31]}$. 但是有机氮和氨氮随时间的变化趋势差异非常明显, 这表明有机氮的矿化过程受水体 DO 、 $\mathrm{pH}$ 和微生物活性的影响较大, 有明显的季节变化, 主要表现为春、冬季矿化速率较高, 夏季矿化速率较 低. 夏季氨氮的来源主要为外源输人和内源底泥释放, 多种因素的共同作用导致了滇池水体氨氮浓度随季 节的复杂变化趋势 ${ }^{[32-33]}$.

\section{3 氮、磷内源释放的贡献及变化}

湖体内氮、磷底泥释放和沉降通量是研究关注的重点. 氨氮和磷酸盐的底泥释放过程有明显的季节特 征, 夏季表现为释放, 冬季则会表现为吸收. 造成这种现象的主要因素除风力扰动外, 最为主要的是底泥氧 化还原条件的改变. 在缺氧条件下, 底泥对氨氮和磷酸盐的吸附效率降低, 从而促进了氨氮和磷酸盐的底泥 释放作用 ${ }^{[17,27,34]}$. 综合考虑颗粒态有机氮的沉降和氨氮、硝态氮的底泥释放作用可得出,氮的水体一底泥界 面交换过程对氮循环总体的贡献表现为释放, 释放量为 $237.6 \mathrm{t} / \mathrm{a}$. 而磷的水体一底泥界面交换过程与氮不 同, 该过程对磷循环总体的贡献表现为沉降, 沉降量为 $173.9 \mathrm{t} / \mathrm{a}$. 但需要注意的是, 底泥磷酸盐的释放随季 节变化非常大, 在藻类暴发期间表现为强烈的释放作用, 日最大值已达到 $24.2 \mathrm{t} / \mathrm{d}$, 是湖体中磷酸盐的主要 来源之一 ${ }^{[35-36]}$. 对比之前针对滇池底泥氮、磷内源释放的研究发现, 利用模拟实验的方法计算氨氮底泥释放 量约为 $1394.1 \sim 6542.2 \mathrm{t} / \mathrm{a}$, 磷酸盐的底泥释放量约为 74.9 380.2 t/a; 本文模型结果中氮、磷的底泥释放量 分别为 2213.05 和 $82.39 \mathrm{t} / \mathrm{a}$, 模型结果与文献中的实验结果范围基本吻合 ${ }^{[37-38]}$. 氮、磷元素间的上述差异主 要与其化学性质相关, 磷循环属于沉积态循环, 其沉降到湖底的磷会经过沉积成岩过程被永久的埋藏在湖 
底; 而氮的化学性质相对活泼, 其沉降到湖底的主要为有机残体, 经微生物分解后会主要以氨氮的形式再次 释放到水体, 使湖体氮浓度维持在较高的水平. 通过对滇池氮、磷元素化学性质以及关键源汇过程通量的分 析,可以为滇池富营养化的治理提供理论和决策支持.

\section{4 结论}

以 IWIND-LR 为计算平台, 核算了滇池外海氮、磷循环通量及其在氮、磷循环整体中的贡献. 研究发现, 通过三维数值模型计算湖泊营养盐存量和关键过程通量以及质量平衡关系相较于传统的质量平衡方法更 为详细, 且可计算反硝化、底泥释放等很难进行实验测定的过程. 其中, 对于 2003 年滇池 TN 的年总输人量 为 $7620.92 \mathrm{t}$, 总输出量为 $7637.31 \mathrm{t}$; TP 的年总输人量为 $450.23 \mathrm{t}$, 总输出量为 $429.57 \mathrm{t}$. 沉降和反硝化则是主 要的氮、磷去除途径, 反硝化占总氮去除量的 $48.99 \%$, 沉降占总磷去除量的 $62.77 \%$. 必须注意的是, 上述的 结果并不能推广为滇池长期的趋势. 今后的研究将集中在 2 个方面: (1) 通过实验手段, 对模型所涉及的关键 过程参数进行测定和核实, 减少模型结果的不确定性和可能存在的异参同效; (2)对滇池进行更长期的模拟, 分析不同水文气象条件和流域负荷条件下的营养盐平衡和通量, 为滇池富营养化控制提供更扎实的理论依 据和技术支持.

\section{5 参考文献}

[ 1 ] Paerl HW, Xu H, Mccarthy MJ et al. Controlling harmful cyanobacterial blooms in a hyper-eutrophic lake (Lake Taihu, China): The need for a dual nutrient (N \& P) management strategy. Water Research, 2011, 45(5) : 1973-1983.

[ 2 ] Schindler DW. Eutrophication and recovery in experimental lakes: Implications for lake management. Science, 1974,184 (4139) : 897-899.

[ 3 ] Qin Boqiang. A review and prospect about the aquatic environment studies in Taihu Lake. J Lake Sci, 1998, 4(4) : 1-9. DOI: 10.18307/1998.0401. [秦伯强. 太湖水环境面临的主要问题、研究动态与初步进展. 湖泊科学, 1998, 4(4) : 1-9. ]

[ 4 ] Conley DJ, Paerl HW, Howarth RW et al. Controlling eutrophication: Nitrogen and phosphorus. Science, 2009, 323 (5917) : 1014-1015.

[ 5 ] Jin Xiangcan. The key scientific problems in lake eutrophication studies. Acta Sci Circum, 2008, 28(1): 21-23. [ 金相灿. 湖泊富营养化研究中的主要科学问题——代 “湖泊富营养化研究” 专栏序言. 环境科学学报, 2008, 28(1): 21-23.]

[ 6 ] Qin Boqiang, Luo Liancong. Changes in eco-environment and causes for lake Taihu, China. Quat Sci, 2004,24(5) : 561568. [ 秦伯强, 罗潋苾. 太湖生态环境演化及其原因分析. 第四纪研究, 2004, 24(5): 561-568.]

[ 7 ] Ruan Jingrong, Cai Qinghua, Liu Jiankang. A phosphorus-phytoplankton dynamic model for Lake Donghu in Wuhan. Acta Hydrobiol Sin, 1988, 12(4) : 289-307. [阮景荣, 蔡庆华, 刘建康. 武汉东湖的磷-浮游植物动态模型. 水生生物学 报, 1988, 12(4): 289-307.]

[ 8 ] Wu Fengchang, Jin Xiangcan, Zhang Runyu et al. Effects and significance of organic nitrogen and phosphorus in the lake aquatic environment. J Lake Sci, 2010,22(1): 1-7.DOI: 10.18307/2010.0101. [吴丰昌, 金相灿, 张润宇等. 论有机 氮磷在湖泊水环境中的作用和重要性. 湖泊科学, 2010, 22(1): 1-7.]

[ 9 ] Carpenter SR, Caraco NF, Correll DL et al. Nonpoint pollution of surface waters with phosphorus and nitrogen. Ecol Appl, $1998,8(3): 559-568$.

[10] Spears BM, Carvalho L, Perkins R et al. Long-term variation and regulation of internal phosphorus loading in loch leven. Hydrobiologia, 2012, 681(1) : 23-33.

[11] Bernhardt ES. Cleaner lakes are dirtier lakes. Science, 2013, 342(6155) : 205-206.

[12] Finlay JC, Small GE, Sterner RW. Human influences on nitrogen removal in lakes. Science, 2013, 342(6155) : 247-250.

[13] Elser JJ, Bracken ME, Cleland EE et al. Global analysis of nitrogen and phosphorus limitation of primary producers in freshwater, marine and terrestrial ecosystems. Ecology Letters, 2007, 10(12) : 1135-1142.

[14] Sondergaard M, Jensen JP, Jeppesen E et al. Seasonal dynamics in the concentrations and retention of phosphorus in shallow danish lakes after reduced loading. Aquatic Ecosystem Health \& Management, 2002, 5(1) : 19-29.

[15] Sondergaard M, Jeppesen E, Lauridsen TL et al. Lake restoration: Successes, failures and long-term effects. Journal of Applied Ecology, 2007, 44(6) : 1095-1105. 
[16] Hanson PC, Bade DL, Carpenter SR et al. Lake metabolism: Relationships with dissolved organic carbon and phosphorus. Limnology Oceanography, 2003, 48(3) : 1112-1119.

[17] Nowlin WH, Evarts JL, Vanni MJ. Release rates and potential fates of nitrogen and phosphorus from sediments in a eutrophic reservoir. Freshwater Biology, 2005, 50(2): 301-322.

[18] Sondergaard M, Bjerring R, Jeppesen E. Persistent internal phosphorus loading during summer in shallow eutrophic lakes. Hydrobiologia , 2013, 710(1) : 95-107.

[19] Kleeberg A, Kozerski HP. Phosphorus release in lake grosser muggelsee and its implications for lake restoration. Hydrobiologia, 1997, 342: 9-26.

[20] Janse JH. A model of nutrient dynamics in shallow lakes in relation to multiple stable states. Hydrobiologia, 1997, 342: $1-8$.

[21] Mooij WM, Trolle D, Jeppesen E et al. Challenges and opportunities for integrating lake ecosystem modelling approaches. Aquatic Ecology, 2010, 44(3): 633-667.

[22] Rowe MD, Kreis RG, Jr Dolan DM. A reactive nitrogen budget for lake michigan. J Gt Lakes Res, 2014, 40 (1): 192-201.

[23] Wang Z, Zou R, Zhu X et al. Predicting lake water quality responses to load reduction: A three-dimensional modeling approach for total maximum daily load. Int J Environ Sci Technol, 2014, 11(2) : 423-436.

[24] He Kediao. Comprehensive evaluation and trend analysis for water quality of Dianchi Lake. Yangtze River, 2012, 43(12): 37-41. [贺克雕. 滇池水质状况综合评价及变化趋势分析. 人民长江, 2012, 43(12) : 37-41.]

[25] Liu Yong, Zou Rui, Guo Huaicheng eds. Intelligent watershed management. Beijing: Science Press, 2012.[刘永, 邹锐, 郭怀成. 智能流域管理研究. 北京:科学出版社, 2012.]

[26] Gao Li, Yang Hao, Zhou Jianmin et al. Sediment burden and release potential of phosphorus to water in Dianchi Lake, China. Acta Sci Circum, 2004, 24(5) : 776-781. [ 高丽, 杨浩, 周健民等. 滇池沉积物磷内负荷及其对水体贡献的研 究. 环境科学学报, 2004, 24(5): 776-781.]

[27] Wang Jianjun, Shen Ji, Zhang Lu et al. Sediment-water nutrient fluxes and the effects of oxygen in Lake Dianchi and Lake Fuxian, Yunnan Province. J Lake Sci, 2010,22( 5) : 640-648.DOI: 10.18307/2010.0503. [王建军, 沈吉, 张路等. 云 南滇池和抚仙湖沉积物-水界面营养盐通量及氧气对其的影响. 湖泊科学, 2010, 22(5): 640-648.]

[28] Xiong Qiang, Jiao Lixin, Wang Shengrui et al. Characteristics and bioavailability of organic phosphorus from different sources of sediments in Dianchi Lake. Environmental Science, 2014,35(11): 4118-4126. [熊强, 焦立新, 王圣瑞等. 滇 池沉积物有机磷垂直分布特征及其生物有效性. 环境科学, 2014,35(11) : 4118-4126.]

[29] Hasegawa T, Okino T. Seasonal variation of denitrification rate in lake suwa sediment. Limnology, 2004, 5(1) : 33-39.

[30] Chen X, Yang L, Xiao L et al. Nitrogen removal by denitrification during cyanobacterial bloom in Lake Taihu. J Freshw Ecol, 2012, 27(2) : 243-258.

[31] Wetzel RG. Limnology: Lake and river ecosystems. Access Online via Elsevier, 2001: 229-266.

[32] Small GE, Cotner JB, Finlay JC et al. Nitrogen transformations at the sediment-water interface across redox gradients in the laurentian great lakes. Hydrobiologia, 2014, 731(1) : 95-108.

[33] Yang L, Choi JH, Hur J. Benthic flux of dissolved organic matter from lake sediment at different redox conditions and the possible effects of biogeochemical processes. Water Research, 2014, 61: 97-107.

[34] Malecki LM, White JR, Reddy KR. Nitrogen and phosphorus flux rates from sediment in the lower st. Johns river estuary. Journal of Environmental Quality, 2004, 33(4) : 1545-1555.

[35] Roy ED, Nguyen NT, Bargu S et al. Internal loading of phosphorus from sediments of lake pontchartrain (louisiana, USA) with implications for eutrophication. Hydrobiologia, 2012, 684(1) : 69-82.

[36] Roy ED, White JR. Nitrate flux into the sediments of a shallow oligohaline estuary during large flood pulses of Mississippi River water. Journal of Environmental Quality, 2012, 41(5) : 1549-1556.

[37] Wang Miao, Yan Hong, Jiao Lixin et al. Characteristics of internal nitrogen loading and influencing factors in Dianchi Lake sediment. Chin Environ Sci, 2015, 35(1): 218-226. [汪永, 严红, 焦立新等. 滇池沉积物氮内源负荷特征及影响因 素. 中国环境科学, $2015,35(1): 218-226$.

[38] Li Bao, Ding Shiming, Fan Chengxin et al. Estimation of releasing fluxes of sediment nitrogen and phosphorus in Fubao Bayin Dianchi Lake. Environmental Science, 2008, 29(1): 114-120. [李宝, 丁士明, 范成新等. 滇池福保湾底泥内源 氮、磷营养盐释放通量估算. 环境科学, 2008, 29(1): 114-120.] 